\title{
Dual-beam confocal light-sheet microscopy via flexible acousto-optic deflector
}

\author{
Vladislav Gavryusev \\ Giuseppe Sancataldo \\ Pietro Ricci \\ Alberto Montalbano \\ Chiara Fornetto \\ Lapo Turrini \\ Annunziatina Laurino \\ Luca Pesce \\ Giuseppe de Vito \\ Natascia Tiso \\ Francesco Vanzi \\ Ludovico Silvestri \\ Francesco S. Pavone
}




\title{
Dual-beam confocal light-sheet microscopy via flexible acousto-optic deflector
}

\author{
Vladislav Gavryusev, ${ }^{\mathrm{a}, \mathrm{b}, \dagger}$ Giuseppe Sancataldo, ${ }^{\mathrm{a}, \mathrm{b}, \dagger}$ Pietro Ricci, ${ }^{\mathrm{a}}$ Alberto Montalbano, ${ }^{\mathrm{c}}$ Chiara Fornetto, ${ }^{\mathrm{a}}$ \\ Lapo Turrini, ${ }^{\mathrm{a}, \mathrm{b}}$ Annunziatina Laurino, ${ }^{\mathrm{a}, \mathrm{b}}$ Luca Pesce, ${ }^{\mathrm{a}, \mathrm{b}}$ Giuseppe de Vito, ${ }^{\mathrm{a}, \mathrm{c}, \mathrm{d}}$ Natascia Tiso, ${ }^{\mathrm{e}}$ Francesco Vanzi, ${ }^{\mathrm{a}, \mathrm{f}}$ \\ Ludovico Silvestri, ${ }^{a, b, d}$ and Francesco S. Pavone ${ }^{a, b, d, *}$ \\ ${ }^{a}$ European Laboratory for Non-Linear Spectroscopy, Sesto Fiorentino, Italy \\ bUniversity of Florence, Department of Physics and Astronomy, Sesto Fiorentino, Italy \\ 'University of Florence, Department of Neuroscience, Psychology, Drug Research and Child Health, Florence, Italy \\ ${ }^{d}$ National Institute of Optics, National Research Council, Sesto Fiorentino, Italy \\ eUniversity of Padova, Department of Biology, Padova, Italy \\ fUniversity of Florence, Department of Biology, Sesto Fiorentino, Italy
}

\begin{abstract}
Confocal detection in digital scanned laser light-sheet fluorescence microscopy (DSLM) has been established as a gold standard method to improve image quality. The selective line detection of a complementary metal-oxide-semiconductor camera (CMOS) working in rolling shutter mode allows the rejection of out-of-focus and scattered light, thus reducing background signal during image formation. Most modern CMOS have two rolling shutters, but usually only a single illuminating beam is used, halving the maximum obtainable frame rate. We report on the capability to recover the full image acquisition rate via dual confocal DSLM by using an acoustooptic deflector. Such a simple solution enables us to independently generate, control and synchronize two beams with the two rolling slits on the camera. We show that the doubling of the imaging speed does not affect the confocal detection high contrast. $\odot$ The Authors. Published by SPIE under a Creative Commons Attribution 4.0 Unported License. Distribution or reproduction of this work in whole or in part requires full attribution of the original publication, including its DOI. [DOI: 10.1117/1.JBO.24.10 .106504]

Keywords: light-sheet microscopy; digital scanned laser light-sheet fluorescence microscopy; confocal detection; acousto-optic deflector; high-throughput microscopy; high contrast; mouse brain; zebrafish brain.
\end{abstract}

Paper 190152LRR received May 13, 2019; accepted for publication Jul. 30, 2019; published online Oct. 31, 2019.

\section{Introduction}

Fluorescence microscopy, due to its combination of molecular specificity and high contrast, has broad application in a wide range of research areas, from cell biology to neuroscience. ${ }^{1,2}$ In particular, light-sheet fluorescence microscopy (LSFM) has become one of the fastest growing techniques for imaging of three-dimensional (3-D) thick samples. ${ }^{3-5}$ By illuminating a single plane of the sample, it provides intrinsic optical sectioning and fast image recording, while minimizing out-of-focus fluorescence background and reducing sample photodamage and photobleaching. In such a microscope, the light-sheet is usually created by means of a cylindrical lens ${ }^{3}$ or by rapidly scanning a Gaussian (or a nondiffracting) beam, as in digital scanned laser light-sheet microscopy (DSLM) ${ }^{6-8}$ The induced fluorescence is imaged through a simple wide-field detection path. However, when 3-D imaging is performed in turbid samples, the illumination light can suffer from scattering effects which lead to spurious background signals that cannot be rejected by the wide-field detection path, effectively degrading the signal-tobackground ratio. Several optimizations of LSFM have been implemented to mitigate or to avoid this effect. In particular, structured illumination (DSLM-SI) ${ }^{9}$ and confocal-like detection $(\mathrm{CLSFM})^{10}$ have been introduced into the DLSM configuration to spatially filter the out-of-focus and scattered light. DSLM-SI requires multiple images and postprocessing, while CLSFM

*Address all correspondence to Francesco S. Pavone, E-mail: pavone@lens .unifi.it

${ }^{\dagger}$ Equal contribution needs only to record line-by-line the fluorescence emitted at each light beam position through a confocal slit placed in the detection path. CLSFM can be implemented either by acquiring an image for each illumination position and then fusing them, ${ }^{7}$ or in a single acquisition by descanning with two additional galvanometric mirrors placed before and after a physical slit, ${ }^{11}$ or by leveraging as a virtual slit the in-built rolling shutter readout mode of scientific complementary metal-oxide-semiconductor (sCMOS) cameras $^{10}$ [Fig. 1(b)]. In the two latter cases, synchronizing the rates and positions of the digital light-sheet and the rolling shutter line-by-line sensor readout is enough to form the confocal image, which is simple and cost-efficient to realize, to the point of stimulating even camera manufacturers to implement a fine control of the rolling shutter parameters in their sensor drivers.

sCMOS cameras typically have two rolling shutters, one for each half of the sensor [Fig. 1(c)], but in most DLSM setups a single beam is scanned by a galvanometric mirror. This requires the use of a single rolling shutter moving from the top to the bottom of the sensor (or vice versa) to obtain confocal detection [Fig. 1(b)], leading to a halving of the maximum frame rate. This limitation becomes important when investigating fast events such as calcium transients ${ }^{12,13}$ or very large volumes, ${ }^{4,9,14-16}$ but can be overcome by generating two independent parallel light-sheets within the field of view (FOV) and by synchronizing them with the two rolling shutters, at the cost of increased complexity. A first such system has been realized using a DLSM ${ }^{17}$ where two focused illuminating beams, coming from two identical but facing each other objectives, scan half the FOV each in opposite directions. However, this can lead to inhomogeneous illumination and counter propagating striping artifacts over the full FOV, 

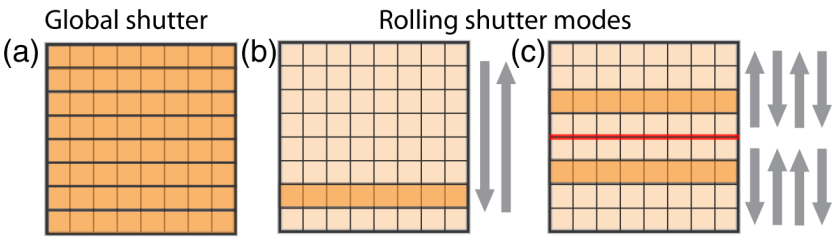

Fig. 1 Image acquisition schemes: (a) in global shutter mode all pixels are exposed at once (orange color), while in (b) single- or (c) dualrolling shutter modes only one or two sets of neighboring pixel rows are concurrently active, before sequentially enabling the next ones in the direction indicated by the arrows. The red line in (c) demarcates the sensor halves.

especially in a turbid sample. A different solution based on a single-sided dual Bessel beam confocal illumination scheme has been reported. ${ }^{18}$ Here, the interline distance between the two beams is fixed and a digital micromirror device has been placed in the detection path to enable the confocal detection on any CMOS camera, with the drawback of additional system complexity and of the camera integrating more dark noise.

Here, we show the advantages provided by acousto-optic deflectors (AODs) for simultaneous dual-beam confocal detection in DLSM. AODs are fast laser beam deflectors that are increasingly applied in the field of high-speed imaging. ${ }^{19,20}$ They are based on a periodically changing refractive index inside a transparent crystal which is induced by propagating sound waves, created by an oscillating piezo at $\mathrm{MHz}$ frequency. The crystal behaves like an optical grating, which diffracts an impinging laser beam. When the piezo of the AOD is driven by a single frequency, it allows to control the deflection and the intensity of a single beam. Interestingly, when the piezo is driven by multiple frequencies, the crystal behaves like a linear combination of gratings, allowing to generate simultaneously different beams from a single one. Each beam can be independently regulated in terms of spatial direction and intensity. Recently, we used this AOD capability to demonstrate the simultaneous generation and control of multiple beams to attenuate striping artifacts present in LSFM images. ${ }^{21}$ In this letter, we report on the use of an AOD in the illumination path of a DLSM to independently generate two Gaussian beams and sweep them across the FOV synchronously with any double rolling shutter readout direction of an sCMOS camera, resulting in a twofold peak frame-rate speed-up in the confocal detection regime, without loss of contrast. We apply this high-speed CLSFM to explore the mouse brain structure with subneuron resolution and to record zebrafish larvae's brain activity, which are typical LSFM applications.

\section{Methods}

\section{1 sCMOS Camera Readout Modes}

To understand the aforementioned performance gain, an overview of the several image acquisition schemes enabled by different sCMOS camera readout modes is beneficial. Figure 1(a) shows the global shutter acquisition mechanism, where every pixel is simultaneously activated to capture the image all at once. Differently, as shown in Figs. 1(b)-1(c), in rolling shutter mode one or few pixel rows are concurrently activated, sweeping across the sensor's matrix and providing a sequential scan of the image. The readout of each pixel row follows the exposure, leading to a line by line temporal shift, even if the exposure time is the same for each line. There exist several sensor readout modes depending on the number and type of rolling shutters implemented by the camera manufacturer. If only one rolling shutter is available, then it can be continuously swept from the top edge to the bottom one or vice versa, as shown in Fig. 1(b). In most state-of-the-art sCMOS cameras, the sensor is divided in two halves that are jointly recorded via two rolling shutters moving from the middle out to the top (or bottom) edges or in the same direction, as depicted in Fig. 1(c), or even in a single sweep from one edge to the other, as if a single shutter was present.

\subsection{Experimental Setup and AOD Operation}

To benefit from the confocal detection modality enabled by the rolling shutter modes on sCMOS cameras, the illuminating beams must be spatially overlapped and synchronized with the moving virtual slit positions on the sensor, as shown in Fig. 2(a). Figure 2(b) displays the schematic of the light-sheet microscope implemented here for single- and dual-confocal detection. The optical architecture is based on a DSLM where the galvo mirror is replaced by an AOD. A visible light beam from a diode laser ( $488 \mathrm{~nm}$, Coherent Sapphire 300) is expanded and collimated by a pair of achromatic lenses (Thorlabs AC254-30-A and AC254150-A). Then, the beam is guided into the AOD (AA Opto Electronic DTSX-400, $\mathrm{TeO}_{2}$, aperture $7.5 \times 7.5 \mathrm{~mm}^{2}$ ) that is driven by a radio frequency (RF) system (four channel signal generator Analog Devices AD9959PCBZ followed by a Minicircuits power combiner ZMSC-2-1W+ and an amplifier ZHL-1-2W-S+). A scanning lens (Thorlabs AC508-200-A, $f_{L}=200 \mathrm{~mm}$ ), placed after the AOD, converts the angular deflection into a lateral displacement of the incident light. The beam is then directed by the excitation tube lens (Thorlabs AC508-100-A, $f_{L}=100 \mathrm{~mm}$ ) to the pupil of an illumination objective lens (Nikon N10X-PF 10X, 0.3 NA, 16 mm WD). The sample, embedded in a cylinder of $1 \%$ agarose gel, was immersed in a water-filled cuvette sized $10 \times 12 \mathrm{~mm}^{2}$. The fluorescence emitted from the sample is collected with an imaging objective (Nikon N10X-PF 10X, 0.3 NA, $16 \mathrm{~mm} \mathrm{WD),} \mathrm{and} \mathrm{a}$ tube lens of focal length of $200 \mathrm{~mm}$ (Thorlabs TTL200-A) creates an image on an sCMOS camera (Hamamatsu Orca-Flash4.0 V3, $2048 \times 2048$ pixels of $6.5 \times 6.5 \mu \mathrm{m}^{2}$ size). The experimental lateral and axial resolutions are 1.5 and $9.0 \mu \mathrm{m}$, respectively, while the light-sheet FWHM waist is $10 \mu \mathrm{m}$ in order to be within one Rayleigh range over the FOV of $1.33 \times 1.33 \mathrm{~mm}^{2}$. The $x y z$ coordinate system is chosen as follows: the light-sheets are created in the $x-y$ plane with the $x$ axis along the beam propagation direction, and the $z$ direction is along the imaging optical axis. A trigger emitted by a National Instruments PXIe6738 card starts the single- or dual-confocal sample illumination and image acquisition processes, by activating one or two RF ramps on the signal generator that governs the AOD deflection of the laser beams and by synchronously moving the virtual slits across the camera sensor, as shown in the timing diagrams in Fig. 3. In the dual diverging or converging rolling shutter modes, if the sample presents a significant background level, cross talk between the two light-sheets may be observable when they overlap in the center of the sensor. To minimize preventively this effect, which does not affect the parallel modes, we tailored the timing and the starting and ending frequencies of the RF ramps to avoid physically overlapping the two light-sheets at any time on the sensor. In Video 1, by imaging a sample of uniform fluorescent $1 \%$ agarose gel immersed in water, we illustrate the AOD capability to generate all the light patterns required to match the available rolling shutter readout modes [shown in Fig. 1(b)-1(c)]. 


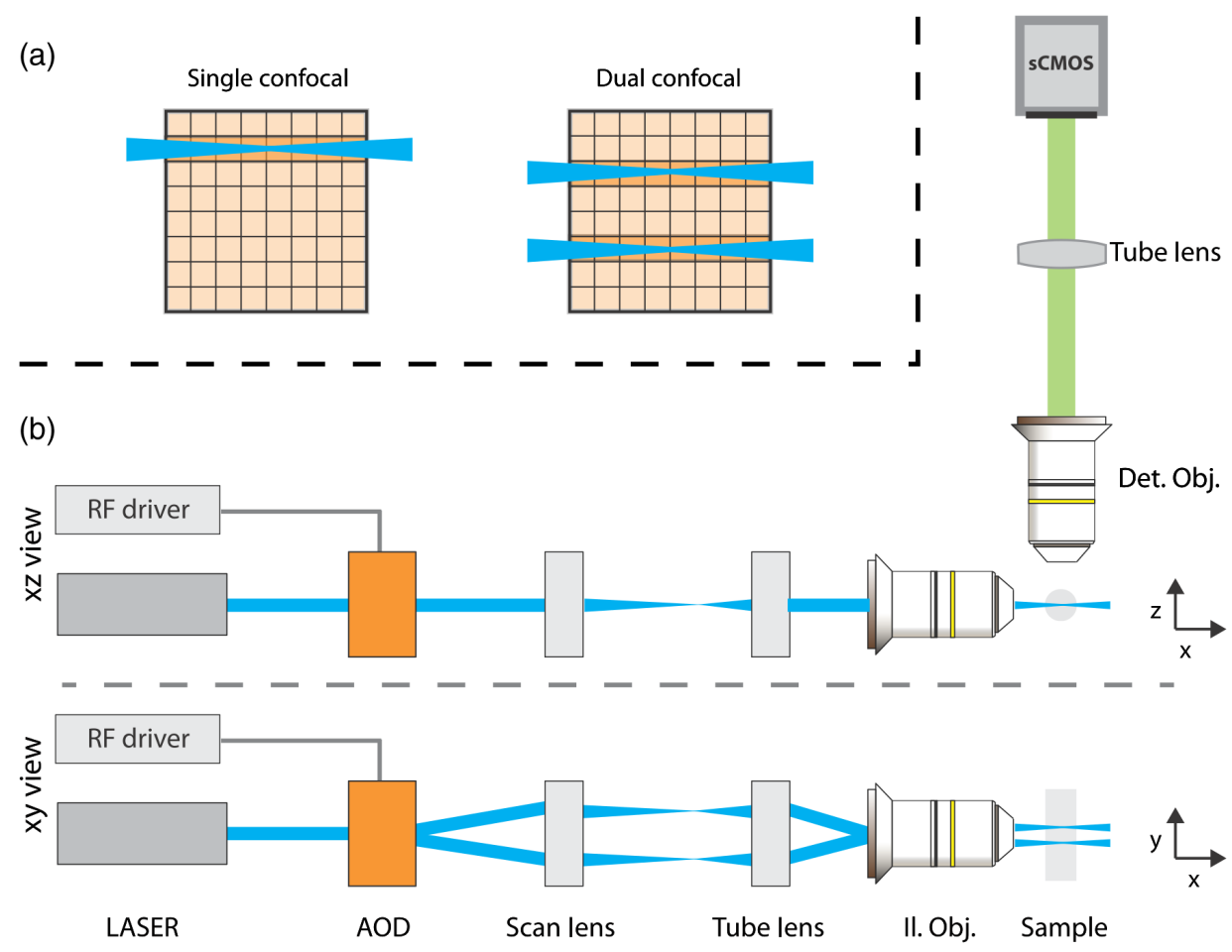

Fig. 2 Schematic of: (a) sCMOS camera operating in single- or dual-rolling shutter mode with the illuminating beam (or beams) matching the position and synchronized with the scan rate of the virtual slit (or slits); (b) the excitation and imaging paths from side and top views.

\subsection{Sample Preparation}

\subsubsection{Mouse}

(a)
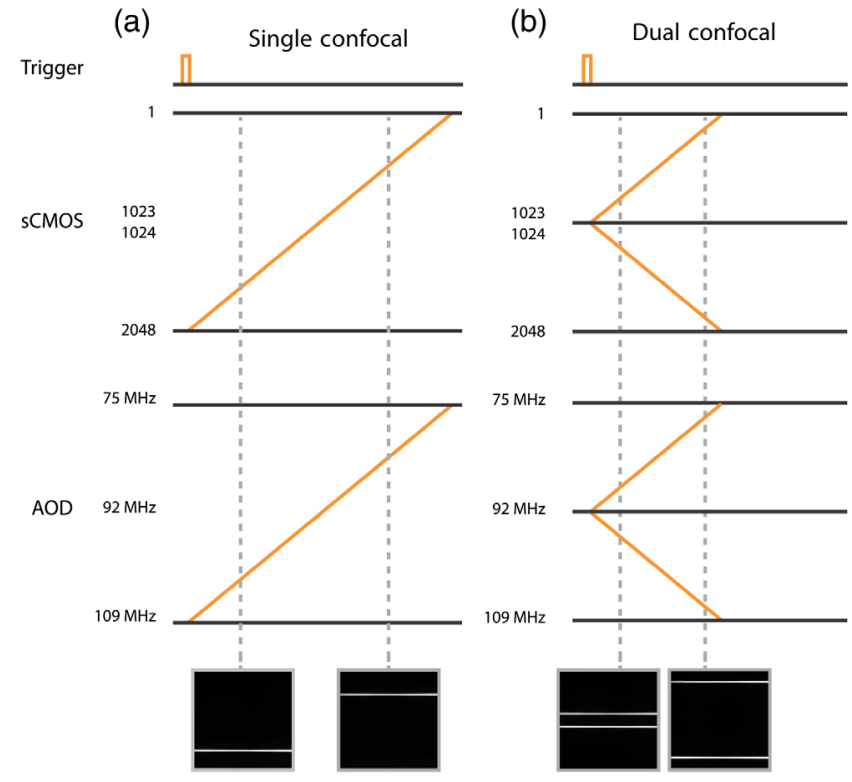

Fig. 3 System timing configuration diagrams for single- (a) and dual-beam (b) confocal illumination. A common trigger starts the camera acquisition and tailored RF ramps on the signal generator that drives the AOD illumination sweep. The image insets are frames from Video 1 (MPEG, 0.1 MB [URL: https://doi.org/10.1117/1.JBO.24.10 .106504.1]), at the times marked by the dotted lines, of a uniform fluorescent $1 \%$ agarose gel in water, imaged with the corresponding rolling shutter readout mode.
One C57B16 mouse was anesthetized with isofluorane and perfused transcardially with ice-cold $4 \%$ paraformaldehyde. The brain was post-fixed overnight at $4^{\circ} \mathrm{C}$ and then cleared using the passive CLARITY technique. ${ }^{24}$ The fixed mouse brain was incubated in hydrogel solution [ $4 \%$ (wt/vol) acrylamide, $0.05 \%$ (wt/vol) bis-acrylamide, $0.25 \%$ (wt/vol) VA044] in 0.01 M PBS at $4^{\circ} \mathrm{C}$ for 3 days. The sample was then degassed and incubated at $37^{\circ} \mathrm{C}$ for $3 \mathrm{~h}$ to allow the hydrogel polymerization. The brain was then extracted from the polymerized gel and incubated in a clearing solution [200 mM sodium borate buffer, $4 \%$ (wt/vol) sodium dodecyl sulfate $(\mathrm{pH} 8.5)]$ at $37^{\circ} \mathrm{C}$ for 1 month while gently shaking. The sample was then washed with PBST $(0.1 \%$ Triton X-100 in $1 \times$ PBS) twice for $24 \mathrm{~h}$ each at room temperature. The cell nuclei were stained by incubation in PBS at RT for $24 \mathrm{~h}$ with Sytox-Green (Thermofisher). The next day, the sample was washed with PBS and imaged. Mouse experiments were carried in accordance with European and Italian law on animal experimentation (D.L. 4 March 2014, no. 26), under authorization no. 790/2016-PR from the Italian Ministry of Health.

\subsubsection{Zebrafish}

Adult and larval zebrafish (Danio rerio) were maintained for breeding at $28^{\circ} \mathrm{C}$ on a $14 \mathrm{~h} / 10 \mathrm{~h}$ light/dark cycle according to standard procedure. Embryos and larvae were raised up to $5 \mathrm{dpf}$ (days postfertilization) in fish water $[150 \mathrm{mg} / \mathrm{L}$ instant ocean, $6.9 \mathrm{mg} / \mathrm{L} \mathrm{NaH} \mathrm{PO}_{4}, 12.5 \mathrm{mg} / \mathrm{L} \mathrm{Na}_{2} \mathrm{HPO}_{4}(\mathrm{pH} \mathrm{7.2)]}$ 
in a Petri dish kept at $28^{\circ} \mathrm{C}$. We used 5 dpf transgenic $T g$ (elavl3: H2B-GCaMP6s) zebrafish larvae ${ }^{22}$ in homozygous albino background to avoid the presence of skin pigments. Each sample was transferred into a $2-\mathrm{mL}$ tube containing $1.5 \% \mathrm{w} / \mathrm{v}$ low gelling temperature agarose (A9414, Sigma) dissolved in fish water, kept at $38^{\circ} \mathrm{C}$, and then introduced into a glass capillary (O.D. $1.5 \mathrm{~mm}$ ) with a pipette, as in Refs. 8 and 25. After gel polymerization, the head portion of larva was extruded from the capillary. To minimize movement artifacts, larvae were preincubated $10 \mathrm{~min}$ in $2 \mathrm{mM}$ d-tubocurarine (T2379, Sigma) dissolved in fish water. The capillary containing the larva was then mounted in a custom-made holder and immersed in the fish water-filled cuvette. Fish rising and experiments were carried in accordance with European and Italian law on animal experimentation (D.L. 4 March 2014, no. 26), under authorization no. 407/2015-PR from the Italian Ministry of Health.

\section{Results}

To test the frame-rate speed-up of our CLSFM in the dual-confocal detection regime over the single one and to verify its impact on the contrast, we imaged samples of mouse brain tissue [Figs. 4(a) and 5] and resting state neural activity in live zebrafish larvae [Fig. 4(b)] in the different rolling shutter readout modes. We chose these types of samples because they are typical examples of structural ${ }^{4,11}$ and functional ${ }^{14,22,23}$ LSFM applications. No qualitative difference between the two detection regimes is observable, moreover, we quantitatively compared them by estimating the contrast in the mouse brain images as in Ref. 9. Each image was normalized by its total intensity, then the standard deviation of the image histogram was calculated and normalized to the single confocal beam case, obtaining an adimensional ratio that quantifies well differences in contrast. The results reported in Table 1 show that the contrast does not (a)

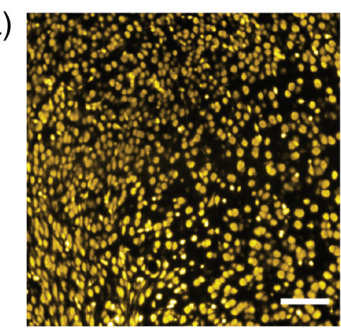

Single CLSFM

(b)
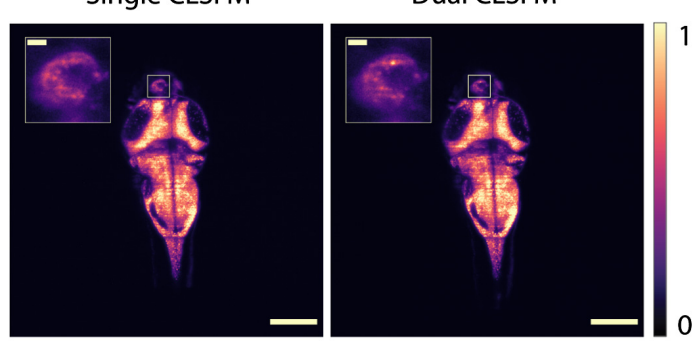

Fig. 4 Representative single (right, top to bottom readout) and dual (left, diverging rolling shutter readout) beam CLSFM full-frame images of (a) cell nuclei in a mouse brain and (b) neuron nuclei in a zebrafish larva brain, respectively, color-coded in yellow and purple. The inset in (b) shows a four times magnified left habenula area within the diencephalon where neural activity can be observed. An extended dual CLSFM zebrafish brain time-lapse recording at $90 \mathrm{fps}$ is shown in Video 2 (MPEG, 2.8 MB [URL: https://doi.org/10.1117/1.JBO.24.10 .106504.2], slowed down to $30 \mathrm{fps}$ ). Scale bar size: $200 \mu \mathrm{m} ; 20 \mu \mathrm{m}$ in the inset. (a)
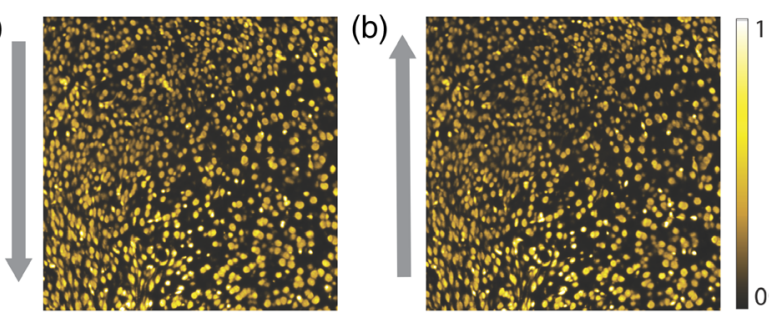

(c)
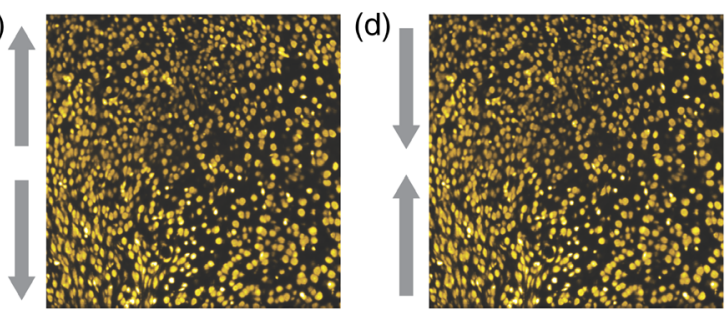

(e)


Fig. 5 Representative (a) and (b) single and (c)-(f) dual beam CLSFM full-frame images of cell nuclei within the same mouse brain cortex area, acquired in the different rolling shutter readout direction modes of the sCMOS camera. No qualitative nor quantitative difference in the image quality is observable.

Table 1 Comparison between single- and dual-beam CLSFM performance.

\begin{tabular}{lccc} 
Modality & Normalized contrast & Frame $_{\text {exp }}(\mathrm{ms})$ & $\mathrm{fps}$ \\
\hline Single beam & $1.000 \pm 0.020$ & 22 & 45 \\
Dual beam & $1.019 \pm 0.015$ & 11 & 90 \\
\hline
\end{tabular}

vary significantly between the two imaging schemes, as expected, since the single row exposure time does not change, while the concurrent readout of the two parts of the camera sensor allows to halve the total exposure time, doubling the frame rate. We verified that no cross talk is observable with the tested highly transparent samples in the sensor center in the diverging [Fig. 5(c)] or converging [Fig. 5(d)] rolling shutter modes. In Video 2, we show an example of a dual CLSFM time-lapse acquisition at 90 frames per second (fps) of zebrafish larvae resting state neural activity, slowed down to $30 \mathrm{fps}$ for presentation convenience. We note that horizontal striping artifacts, mainly due to blood flow, are present in both Video 2 and Fig. 4(b).

\section{Conclusion}

In summary, we have implemented an AOD-based dual Gaussian beam excitation system capable of single- and dualvirtual slit confocal LSFM. AODs allow inertia-free $\mathrm{MHz}$ scan rates and the generation of multiple sheets with independent spatial and amplitude control, ${ }^{21}$ which can be easily synchronized with any single- or dual-rolling shutter readout mode of an 
sCMOS camera. We have demonstrated that the dual-confocal detection regime achieves a twofold improvement in imaging speed, in respect to traditional confocal LSFM, without any negative impact on the contrast, at the very least in the case of samples with small background levels such as optically cleared tissues or zebrafish larvae. Since the AOD behaves here like a galvo mirror, but has smaller scan angles, our method would work well with objectives that have higher magnification and resolution, but an equal or smaller FOV than ours. It would operate even with higher FOV objectives as long as the desired lightsheet waist is obtainable by selecting suitable scan and tube lenses and input beam size. A further improvement of the image quality may be achieved by attenuating striping artifacts, which would require adding a dual-sided illumination, a beam pivoting system $^{21}$ or switching to Bessel beam illumination ${ }^{8,18}$ by introducing after the AOD two suitable axicons or a spatial light modulator. Our method may prove useful for high throughput imaging of large tissue volumes ${ }^{4,9,14-16}$ and live biological studies with high temporal resolution. ${ }^{12,13,23}$

\section{Disclosures}

The authors declare that there are no conflicts of interest related to this paper.

\section{Acknowledgments}

This project has received funding from the European Union's Horizon 2020 Framework Programme for Research and Innovation under Grant Agreement No. 654148 (LaserlabEurope), specific Grant Agreement No. 720270 (Human Brain Project SGA1), No. 785907 (Human Brain Project SGA2), and Marie Skłodowska-Curie Grant Agreement No. 793849 (MesoBrainMicr), from the General Hospital Corporation Center of the National Institutes of Health under award number 1U01MH117023-01, from the H2020 EXCELLENT SCIENCE-European Research Council (ERC) under Grant Agreement ID No. 692943 (BrainBIT) and from the Italian Ministry for Education in the framework of Eurobioimaging (ESFRI research infrastructure)—Advanced Light Microscopy Italian Node. The authors are grateful to Mr. Riccardo Ballerini from LENS mechanical workshop for technical assistance.

\section{References}

1. J. W. Lichtman and J. A. Conchello, "Fluorescence microscopy," Nat. Methods 2(12), 910-919 (2005).

2. N. Ji, J. Freeman, and S. L. Smith, "Technologies for imaging neural activity in large volumes," Nat. Neurosci. 19(9), 1154-1164 (2016).

3. J. Huisken et al., "Optical sectioning deep inside live embryos by selective plane illumination microscopy," Science 305(5686), 1007-1009 (2004).

4. P. J. Keller and H.-U. Dodt, "Light sheet microscopy of living or cleared specimens," Curr. Opin. Neurobiol. 22(1), 138-143 (2011).

5. R. M. Power and J. Huisken, "A guide to light-sheet fluorescence microscopy for multiscale imaging," Nat. Methods 14(4), 360-373 (2017).

6. P. J. Keller et al., "Reconstruction of zebrafish early embryonic development by scanned light sheet microscopy," Science 322(5904), 1065-1069 (2008).

7. F. O. Fahrbach and A. Rohrbach, "Propagation stability of self-reconstructing Bessel beams enables contrast-enhanced imaging in thick media," Nat. Commun. 3(632), 632 (2012).

8. M. C. Müllenbroich et al., "Bessel beam illumination reduces random and systematic errors in quantitative functional studies using light-sheet microscopy," Front. Cell. Neurosci. 12, 315 (2018).
9. P. J. Keller et al., "Fast, high-contrast imaging of animal development with scanned light sheet-based structured-illumination microscopy," Nat. Methods, 7(8), 637-642 (2010).

10. E. Baumgart and U. Kubitscheck, "Scanned light sheet microscopy with confocal slit detection," Opt. Express 20(19), 21805-21814 (2012).

11. L. Silvestri et al., "Confocal light sheet microscopy: micron-scale neuroanatomy of the entire mouse brain," Opt. Express 20(18), 20582-20598 (2012).

12. D. Storace et al., "Toward better genetically encoded sensors of membrane potential," Trends Neurosci. 39(5), 277-289 (2016).

13. Y. Bando et al., "Comparative evaluation of genetically encoded voltage indicators," Cell Rep. 26(3), 802-813 (2019).

14. M. B. Ahrens et al., "Whole-brain functional imaging at cellular resolution using light-sheet microscopy," Nat. Methods 10(5), 413-420 (2013).

15. L. Silvestri et al., "Clearing of fixed tissue: a review from a microscopist's perspective," J. Biomed. Opt. 21(8), 081205 (2016).

16. G. de Medeiros et al., "Confocal multiview light-sheet microscopy," Nat. Commun. 6, 8881 (2015).

17. Z. Yang et al., "Dual-slit confocal light sheet microscopy for in vivo whole-brain imaging of zebrafish," Biomed. Opt. Express 6(5), 1797-1811 (2015)

18. P. Zhang et al., "Light-sheet microscopy by confocal line scanning of dual-Bessel beams," J. Biomed. Opt. 21(10), 100502 (2016).

19. G. D. Reddy and P. Saggau, "Fast three-dimensional laser scanning scheme using acousto-optic deflectors," J. Biomed. Opt. 10(6), 064038 (2005).

20. M. Duocastella et al., "Fast inertia-free volumetric light-sheet microscope," ACS Photonics 4(7), 1797-1804 (2017).

21. G. Sancataldo et al., "Flexible multi-beam light-sheet fluorescence microscope for live imaging without striping artifacts," Front. Neuroanat. 13, 7 (2019).

22. N. Vladimirov et al., "Light-sheet functional imaging in fictively behaving zebrafish," Nat. Methods 11(9), 883-884 (2014).

23. G. Sancataldo et al., "Advanced fluorescence microscopy for in vivo imaging of neuronal activity," Optica 6(6), 758-765 (2019).

24. K. Chung et al., "Structural and molecular interrogation of intact biological systems," Nature 497(7449), 332-337 (2013).

25. L. Turrini et al., "Optical mapping of neuronal activity during seizures in zebrafish," Sci. Rep. 7(1), 3025 (2017).

Vladislav Gavryusev received his MS degree in physics at the University of Florence in 2012. He then moved to the University of Heidelberg, where he obtained his PhD in physics in 2016. Since 2017, he has been a postdoctoral researcher in biophysics at the European Laboratory for Non-Linear Spectroscopy and at the University of Florence. His research activity is focused on light-sheet microscopy of cleared specimens to map their structure and to study the effects of neuropathologies.

Giuseppe Sancataldo received his MS degree in physics in 2013 at the University of Palermo. He then moved to the Italian Institute of Technology in Genoa, where he obtained his $\mathrm{PhD}$ in bioengineering and robotics. Since 2017, he is a postdoctoral researcher in biophysics at the European Laboratory for Non-Linear Spectroscopy and at the University of Florence. His research activity is focused on advanced fluorescence microscopy for fast imaging of large biological samples.

Pietro Ricci received his MS degree in experimental physics at the University of Trento in 2018. He then moved to the University of Florence, where he is getting a $\mathrm{PhD}$ in atomic and molecular photonics. Since November 2018, he has been working at the European Laboratory for Non-Linear Spectroscopy. His research activity is focused on light-sheet microscopy of zebrafish specimens and multi-beam illumination with acousto-optic deflectors.

Alberto Montalbano received his MS degree in neuroscience in 2008 from the University of Trieste. He then obtained his PhD in neuroscience and cognitive science in 2012. Since 2012, he has been a postdoctoral researcher in neuropharmacology at the NEUROFARBA Department of the University of Florence. His research activity is mainly focused on the electrophysiological study of the physiology and pharmacology of the serotonin system. 
Lapo Turrini received his MS degree in biology at the University of Florence in 2015. He then obtained his international $\mathrm{PhD}$ in atomic and molecular photonics at the European Laboratory for Non-linear Spectroscopy in 2019. Currently, he is a research fellow at the Department of Physics and Astronomy at the University of Florence. His research activity is focused on whole-brain functional imaging of zebrafish neuronal activity by light-sheet microscopy.

Annunziatina Laurino obtained her MS degree in pharmaceutical chemistry and technology in 2012 and her PhD in drug areas and innovative treatments in 2017 at the University of Florence, following-up with a one-year post-doc. Since 2018, she works as post-doc at the European Laboratory for Non-Linear Spectroscopy. Her research activity focuses on the three-dimensional reconstruction of human brain neuronal networks using light-sheet and two-photon fluorescence microscopies. She received the Young Researchers SIF Farmindustria 2018 Award.

Giuseppe de Vito obtained his $\mathrm{PhD}$ in molecular biophysics in 2016 at Scuola Normale Superiore (Pisa) in collaboration with the Italian Institute of Technology, and a postgraduate diploma in advanced biostatistics at University of Padua. Former junior specialist at University of California-Irvine, in $\mathbf{2 0 1 7}$ he become fixed-term researcher at the Italian National Research Council, before moving in 2019 to the University of Florence. His research focuses on nonlinear optical microscopy of biological samples with different modalities.
Natascia Tiso received her graduation in biological sciences at the University of Padova in 1994 and her PhD in genetic sciences at the University of Bologna, Italy, in 1998. She has been a visiting scientist at the University of Toronto (Canada), at the MPI of Tuebingen, and at the University of Freiburg (Germany). Currently, she is an associate professor in applied biology and vice-director of the Zebrafish Centre at the University of Padova.

Ludovico Silvestri received his MS degree in physics in 2008 at the University of Pisa and his $\mathrm{PhD}$ in atomic and molecular spectroscopy at the University of Florence in 2012. He worked as postdoc at LENS until 2014, then as Researcher at the National Institute of Optics until 2019. Currently, he is an assistant professor at the Physics and Astronomy Department of the University of Florence. His research activity is focused on light-sheet microscopy of cleared specimens.

Francesco S. Pavone is developing new microscopy techniques for high-resolution and high-sensitivity imaging and for laser manipulation purposes. These techniques have been applied to single-molecule biophysics, single-cell imaging, and optical manipulation. Tissue imaging is another active research area, where nonlinear optical techniques have been applied for skin and neural tissue imaging, also in vivo. Currently, he is the director of the European Laboratory for Non-Linear Spectroscopy in Florence.

Biographies for the other authors are not available. 